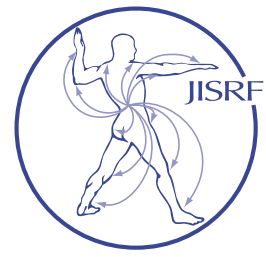

\title{
Conversion Knee Arthroplasty Using a Rotating Hinge as a Salvage Prosthesis Following Periprosthetic Joint Infection and Ligamentous Insufficiency: A Case Report
}

Minter $J^{1}$

\section{Abstract}

Background: In the event of a complex revision TKA in which there is extensor mechanism involvement and ligamentous instability or insufficiency, non-linked levels of constraint may not be adequate for achieving restoration of patient function. Total knee arthroplasty devices that incorporate a linked level of constraint are successful alternatives to unlinked devices (PS and PS-Constrained) in this clinical context.

Case Presentation: We present the case of a 62 yearold male patient that required a non-articulating knee fusion and multiple total knee arthroplasty revisions in conjunction with a ruptured and repaired extensor mechanism, ligamentous instability, bone loss and periprosthetic joint infection. (Revision knee prosthesis that includes a increasing degree of nodularity and physical constraint). The subsequent risk factors associated with the loss of bone and ligamentous insufficiency required performing conversion arthroplasty with a knee prosthesis that includes an increasing degree of modularity and physical constraint not commonly used in revision total knee arthroplasty.

Discussion: The authors report on a patient who underwent multiple operative procedures, we outline the step wise decision making progression that lead to the successful eradication of the PJI and reimplant device strategy based on the confounding factors presented. We assess the use of revision TKA systems that offer extreme degrees of constraint which should be considered in complex revision knee revision procedures.

\section{Background}

The goals of primary total knee arthroplasty (TKA) include relief of pain, restoration of function and establishment of durable long term prosthesis to patient composite. Various factors that contribute to the long-term success of primary TKA include patient co-morbidities, patient compliance, surgical technique and arthroplasty implant design. [1 $\underline{-5}]$ In the event of failure, it is important there is assessment and understanding of the factors resulting in the failure, these include patient co-morbidities, implant loosening, bone loss, knee ligament laxity and periprosthetic joint infection (PJI) [ㅁ-ㄹ]

For aseptical loose TKA requiring revision, without ligamentous involvement or significant bone loss, there are many non-linked device options. [10] In revision TKA in which there is ligamentous instability or insufficiency, loss of extensor mechanism or bone loss non-linked levels of

Keywords: Complex Revision TKA, Rotating Hinge TKA, NonArticulating Fusion, Salvage TKA

Level of Evidence: AAOS Therapeutic Level IV

Educational Value \& Significance: JISRF Level C 
constraint may not be adequate for achieving restoration of function. $[\underline{7}, \underline{8}]$ Total knee arthroplasty devices that incorporate a linked level of constraint are successful alternatives to unlinked devices (PS and PS-Constrained). [ $[\underline{\underline{8}}, \underline{8}]$ We describe a case in which a patient underwent revision reconstruction with a rotating hinged TKA system to manage ligamentous and extensor mechanism deficiency.

\section{Case Presentation}

Prior to this case presentation all patient information was de-identified or removed as per the Health Insurance Portability and Accountability Act (HIPAA, 1996) provisions for safeguarding medical information. In 2007, a 62 year-old male patient underwent a right posterior-stabilized (PS) primary TKA for the management of primary osteoarthritis. The patient reported pain relief and improved function for the first 8 post-operative years . In2015, the patient reported increased pain and right knee instability during start-up, standing and walking without a history of specific injury. X-rays and bone scan revealed tibial component varus malalignment and radiolucency consistent with tibial component loosening. Physical exam revealed knee range of motion (ROM) was 5-120 degrees, there was significant AP laxity (drawer test) but the knee was stable under varus valgus stress. PJI was ruled out by laboratory investigations.

The patient underwent right knee revision TKA, 3 weeks post revision the knee "buckled" whilst the patient was transferring to bed and the patient suffered a fall. The patient experienced pain and loss of extension. X-rays revealed a "high-riding" patella (Figures $1 \mathrm{~A} \&$ 1B). An MRI was ordered but was inconclusive due to metal artifact. Ultrasound and MRI were inconclusive. Operative exploration revealed an extensor mechanism tear which was repaired and the patient was managed partial weight bearing in a locked knee brace in full extension for 4 weeks. Post operative radiographs revealed a well placed posterior stabilized (PS) revision right knee without evidence of patella alta.

Seven weeks post revision the patient was weaning out of the knee brace with 10-90 ROM. The patient presented to a local Emergency Room (ER) with severe back pain. The patient had a prior history of severe lumbar degenerative disc disease (DDD) with prior posterior instrumented interbody fusion that resulted in an early post spine surgery right foot-drop. Following the acute ER presentation the patient was commenced on steroids, muscle relaxing medication, NSAID and was referred to spinal physician. A subsequent MRI revealed evidence of lumbar osteomy-

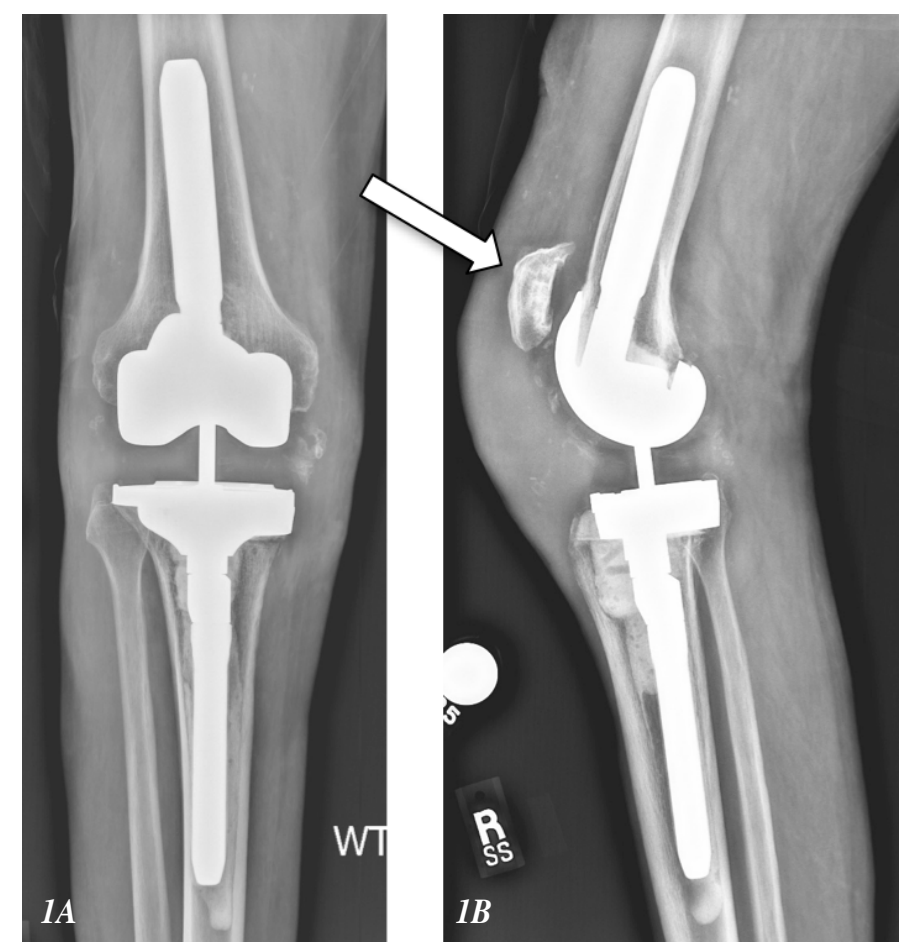

Figures $1 A \& 1 B$. AP and Lateral radiographs following revision TKA and fall resulting in possible quadriceps rupture and subsequent radiographic evidence of patella alta (arrow).

elitis and discitis. The patient was hospitalised for treatment and during admission a diagnosis of septic arthritis of the right knee was confirmed after right knee aspiration grew staph-epidermidis. The patient was treated with a full course of vancomycin and rifampicin.

Two months post septic arthritis the patient presented to the lead author's office for a second opinion. Physical exam revealed active ROM 40-120 degrees, passive ROM 0-120 degrees. There was Grade II instability through ROM, and there was a palpable defect of the patella tendon. Laboratory results showed elevated CRP at 9.41 (0.5 high normal) and an ESR of 99 (20 high normal). Synovasure $^{\circledR}$ (Zimmer Biomet, Warsaw, IN) testing of the knee aspiration was positive for infection with associated count of 5,391 total nucleated cells and 89.2 neutrophils with no bacterial growth noted at 7-days. Two stage revision surgery was scheduled with complete hardware removal and temporary placement of antibiotic impregnated spacer with planned temporary arthrodesis. The arthrodesis procedure was performed utilizing a double set-up technique and the patient exhibited pan-articular grade IV osteomyelitis of the knee. [11] The surgical procedure included a total synovectomy, radical resection of the distal femur and proximal tibia, and the patellar tendon was debrided back to a healthy tissue border. A LINK ${ }^{\circledR}$ Endo-Model ${ }^{\circledR}$ Knee Fusion Nail (Waldemar-LINK GmbH \& Co. KG, Hamburg, Germany) was implanted with antibiotic impregnated ce- 

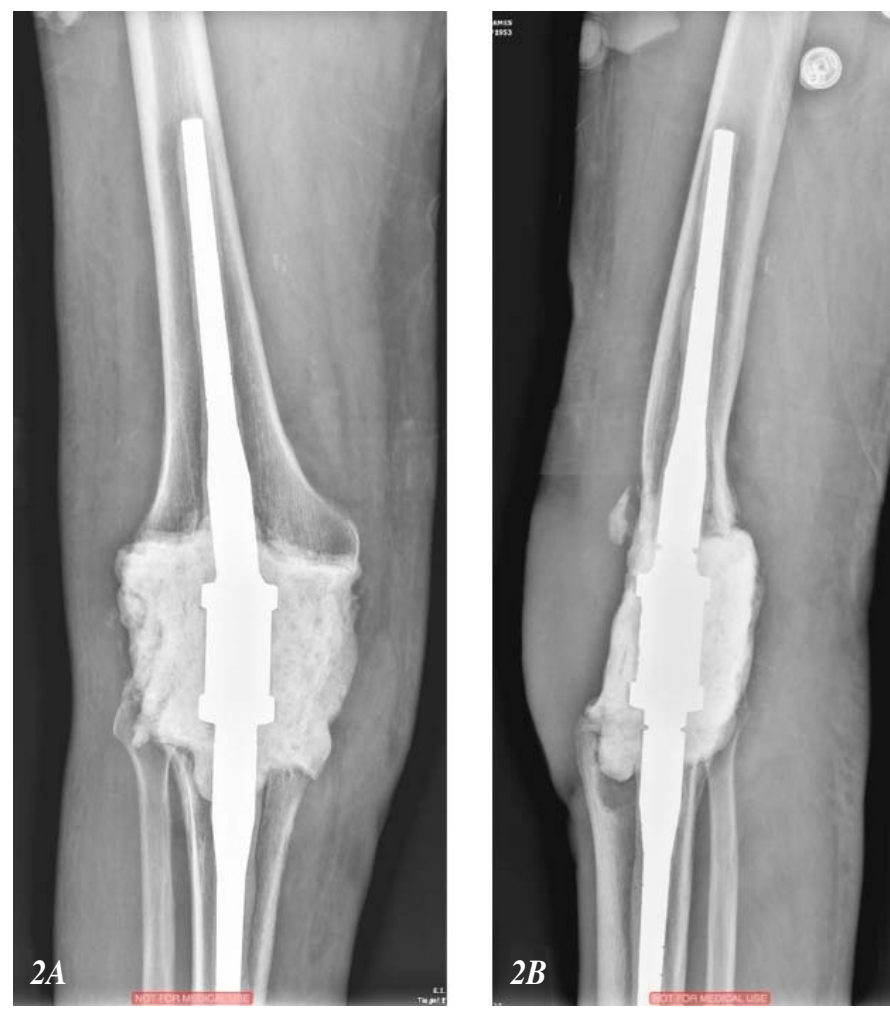

Figures $2 A \& 2 B . A P \&$ Lateral radiographs following fusion utilizing the LINK Endo-Model@ Knee Fusion Nail, implanted with antibiotic impregnated cement and antibiotic beads within the intramedullary canals.

ment and antibiotic beads within the intramedullary canals (Osteoboost ${ }^{\mathrm{TM}}$, Osteoremedies, Inc., Memphis, TN) (Figures $2 \mathrm{~A} \& 2 \mathrm{~B}$ ). Post-operative intravenous (IV) antibiotic which included Vancomycin and Rifampicin treatment that was co-ordinated with Infectious Disease Service.

Two months following the first stage operation the patient completed a full course of antibiotic therapy and two, staged aspirations of the knee were performed to rule out infection (1-month following completion of IV antibiotics, and prior to removal of the arthrodesis nail and revision TKA re-implantation). Both staged Synovasure ${ }^{\circledR}$ aspiration results were negative for infection and the patient was scheduled for revision/reimplantation surgery in November 2016.

At second stage a limb salvage procedure utilizing the LINK ${ }^{\circledR}$ Megasystem- $\mathrm{C}^{\circledR}$ rotating-hinge, segmental replacement knee system (Waldemar-LINK GmbH \& Co. KG, Hamburg, Germany) was performed (Figures 3A\& 3B). A Triathlon ${ }^{\circledR}$ TS Cone (Stryker, Kalamazoo, MI) was incorporated into the reconstruction due to significant tibial bone loss. Extensor mechanism repair utilized a Marlex ${ }^{\circledR}$ graft (C.R. Bard, Warwick, RI) (Brown-Hansen technique), with an associated medial gastrocnemius and split thickness skin graft flap closure as per the plastic surgery consult. Post-operatively, the patient was placed in
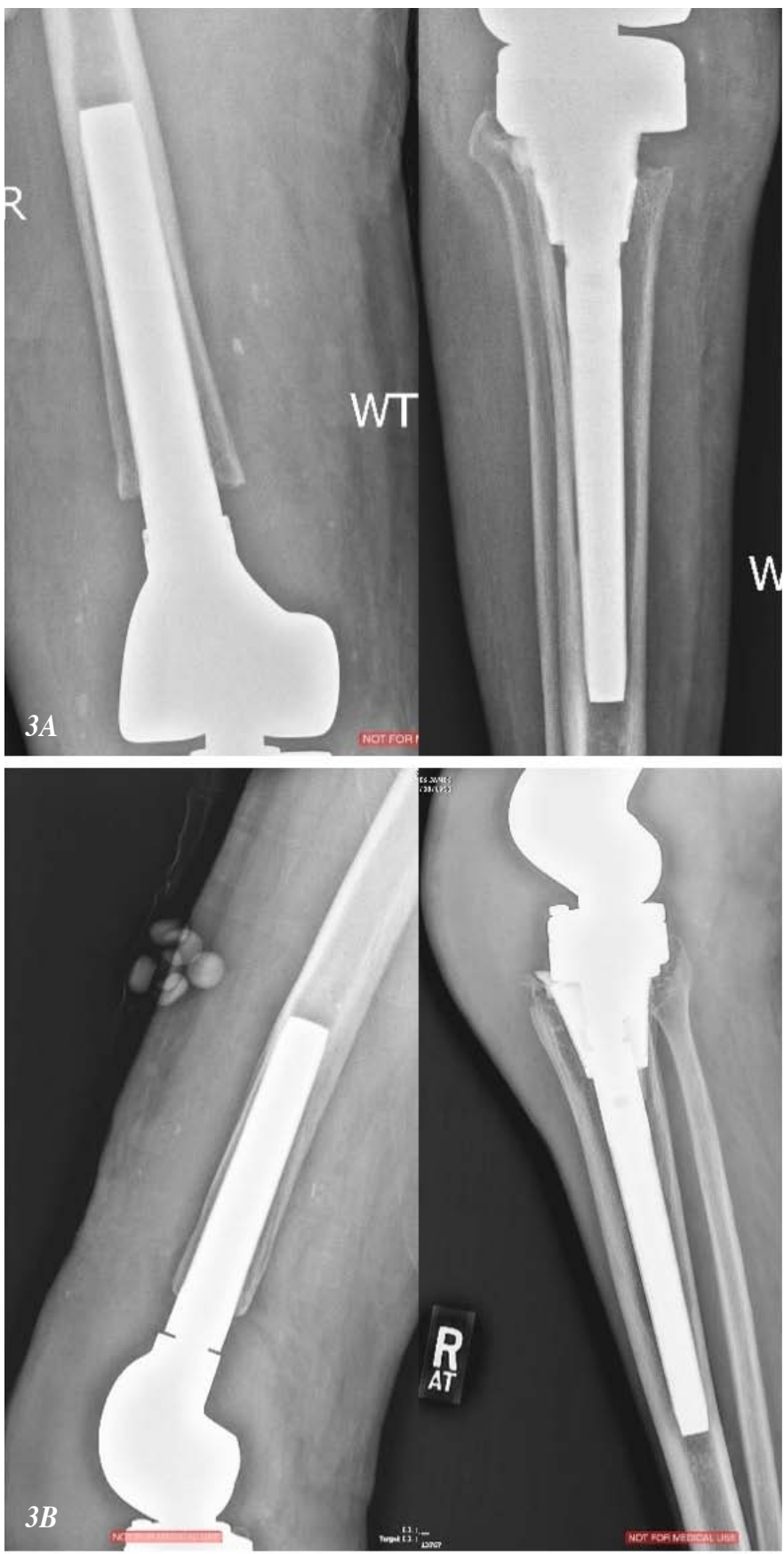

Figures $3 A \& 3 B$. Early post-operative AP and Lateral radiographs following conversion TKA from the antibiotic spacer to the LINK ${ }^{\circledR}$ Megasystem-C rotating-hinge, segmental replacement knee system. Images including the titanium tibial cone to correct for significant bone loss.

a long leg cast for 2 months and allowed only toe-touch weight-bearing. Three months post reimplantation (February 2017), long leg cast was removed, the patient reported minimal pain, physical exam showed no effusion and the patient was able to straight leg raise without extensor lag. A long leg hinged brace initially set at 0-10 degrees flexion was applied with instructions for the patient to remain in brace 24 hours a day and to increase flexion 
by 10 degrees per week. This was performed with no formal physical therapy scheduled. Within six-weeks (March 2017), the patient returned to clinic with only occasional discomfort at the extremes of flexion, however the patient required narcotic pain relief for chronic lumbar pain. Physical exam revealed 0-75 ROM and the patient was advised to transfer to a shorter hinged knee brace upon returning to work. A home exercise program which included quadriceps strengthening and range of motion exercises was encouraged. At most recent follow up the patient's ROM was 0-100 degrees with ambulation up to one mile and stair climbing with alternating gait.

\section{Discussion}

Multiple factors contribute to the overall success or failure of primary and revision TKA. In revision TKA implant options include implant modularity and level of constraint which can assist in addressing various degrees of bone loss, ligamentous insufficiency or instability and extensor mechanism derangement. With the case the authors present revision was required for a combination of factors including component composite failure (aseptic loosening), patient compliance (fall and quadriceps rupture) and patient co-morbidities (infection and previous spine surgery).

This patient experienced knee "buckling" following revision surgery for aseptic loosening. The authors anticipate the patient suffered a patella tendon rupture due to the radiographic evidence of patella alta and PJI was confirmed using the Synovasure PJI test panel. Synovasure was developed to detect infection using local synovial fluid and includes a battery of tests including alpha defensin, CRP and hemoglobin in synovial fluid. $[\underline{14}, \underline{15}]$ The current standard of care for deep infection is considered to be twostage revision arthroplasty and the use of antibiotic-impregnated bone-cement spacers. $[16,17]$ However, in this case the authors took the extra measure to assess on two occasions for any evidence of infection following discontinuation of the IV antibiotics, prior to conversion of the arthrodesis to TKA.

In periprosthetic joint infections with minimal bone loss or soft-tissue insufficiency the patients can be successfully treated utilizing static or mobile antibiotic impregnated spacers and a two-stage TKA re-implantation. $[16,17]$ However, cases involving significant bone loss or soft-tissue insufficiency, an arthrodesis nail with antibiotic impregnated cement is preferred to allow the limb to be brought out to length to avoid soft-tissue contraction and for later placement of the limb salvage TKA system. [1820] In the authors' case the finding of no serviceable ex- tensor mechanism made the use of the fusion nail necessary. The argument for the use of a non-articulating spacer was discussed at the recent International Consensus Conference on PJI (2013), and it was agreed upon that only the technical feasibility of the procedure to be the contraindication for the selection of non-articulating versus articulating spacers. [21] Furthermore, it was the opinion of the consensus group that "patients with massive bone loss and/ or lack of integrity of soft-tissues or ligamentous restraint, strong consideration should be given to the use of non-articulating spacers.'[21]

In patients with ligamentous deficiency, combined with extensor mechanism disruption the pathology taxes the use of non-linked, lower constrained implants leading to overall knee instability and failure. $[7, \underline{8}, \underline{22}]$ In the case the authors report there was significant ligament instability, extensor mechanism insufficiency, bone loss and PJI. The LINK Megasystem-C rotating hinge knee system offers a physically "linked" constraint which is appropriate to manage these deficiencies. Following revision TKA using the LINK Megasystem-C rotating hinge, the patient postoperatively presented with significant improvement in the reduction of pain and restoration of function. Sanguineti, et al reported $93.3 \%$ survival following complex primary and revision cases using the LINK Endo-Model rotating hinge in 45 patients at an average follow-up of 42.2 months. [22] Despite the high degree of constraint, the incidence of aseptic loosening is reported to be minimal, reflecting the manufacturer's claim of force dampening across the rotating hinge mechanism. $[\underline{7}, \underline{8}, \underline{22}, \underline{23}]$

In conclusion, in a complex knee arthroplasty revision scenario careful assessment of the reasons for the adverse outcome must be undertaken. Treatment of PJI prior to revision and confirmation of infection resolution are necessary to avoid further chronic sepsis. The use of revision TKA systems that offer high degrees of constraint should be considered to address issues of multiple ligament insufficiency, extensor mechanism disruption and bone loss.

The authors acknowledge the limitation of case reports of complex procedures which represent a small number of overall arthroplasty cases in the community. Our intent is to report on the sequential degradation on patient's knee that underwent multiple procedures and outline the stepwise progression of treatment that led to successful eradication of PJI and successful re-implantation of a highly constrained linked knee arthroplasty device appropriate for limb salvage procedures.

\section{Acknowledgements}

The author would like to thank Robert W. Eberle for his help in writing this article. 


\section{References}

1. Ong, K.L., et al., Risk of subsequent revision after primary and revision total joint arthroplasty. Clin Orthop Relat Res, 2010. 468(11): p. 3070-6.

2. Lai, K., et al., Presence of medical comorbidities in patients with infected primary hip or knee arthroplasties. J Arthroplasty, 2007. 22(5): p. 651-6.

3. Bozic, K.J., et al., Patient-related risk factors for periprosthetic joint infection and postoperative mortality following total hip arthroplasty in Medicare patients. J Bone Joint Surg Am, 2012. 94(9): p. 794-800.

4. Victor, J., et al., Total knee arthroplasty at 15-17 years: does implant design affect outcome? Int Orthop, 2014. 38(2): p. 235-41.

5. Uvehammer, J., Knee joint kinematics, fixation and function related to joint area design in total knee arthroplasty. Acta Orthop Scand Suppl, 2001. 72(299): p. 1-52.

6. Firestone, T.P. and R.W. Eberle, Surgical management of symptomatic instability following failed primary total knee replacement. J Bone Joint Surg Am, 2006. 88 Suppl 4: p. 80-4

7. Barrack, R.L., et al., The use of a modular rotating hinge component in salvage revision total knee arthroplasty. J Arthroplasty, 2000. 15(7): p. 858-66.

8. Lombardi, A.V., Jr., et al., Rotating hinge prosthesis in revision total knee arthroplasty: indications and results. Surg Technol Int, 1997. 6: p. 379-82.

9. Parvizi, J., et al., The 2018 Definition of Periprosthetic Hip and Knee Infection: An Evidence-Based and Validated Criteria. J Arthroplasty, 2018. 33(5): p. 1309$1314 \mathrm{e} 2$.

10. Bozic, K.J., et al., The epidemiology of revision total knee arthroplasty in the United States. Clin Orthop Relat Res, 2010. 468(1): p. 45-51.

11. Waterman JA, M.J. Minter, Ghattas PJ, Green BM, The Impact of use of Double Set-up on Infection Rates in Revision Total Knee Replacement and Limb Salvage Procedures. Reconstructive Review, 2015. 5(1).

12. Paulos, L.E., et al., The biomechanics of lateral knee bracing. Part I: Response of the valgus restraints to loading. Am J Sports Med, 1987. 15(5): p. 419-29.

13. France, E.P., et al., The biomechanics of lateral knee bracing. Part II: Impact response of the braced knee. Am J Sports Med, 1987. 15(5): p. 430-8.

14. Marson, B.A., et al.,Alpha-defensin and the Synovasure lateral flow device for the diagnosis of prosthetic joint infection. Bone Joint J, 2018. 100-B(6): p. 703-711.

15. de Saint Vincent, B., et al., Diagnostic accuracy of the alpha defensin lateral flow device (Synovasure) for periprosthetic infections in microbiologically complex situations: A study of 42 cases in a French referral centre. Orthop Traumatol Surg Res, 2018. 104(4): p. 427-431.

16. Guild, G.N., 3rd, B. Wu, and G.R. Scuderi, Articulating vs. Static antibiotic impregnated spacers in revision total knee arthroplasty for sepsis. A systematic review. J Arthroplasty, 2014. 29(3): p. 558-63.

17. Anagnostakos, K. and C. Meyer, Antibiotic Elution from Hip and Knee Acrylic Bone Cement Spacers: A Systematic Review. Biomed Res Int, 2017. 2017: p. 4657874.

18. Cho, S.H., et al., Two-stage conversion of fused knee to total knee arthroplasty. J Arthroplasty, 2008. 23(3): p. 476-9.

19. Henkel, T.R., et al., Total knee arthroplasty after formal knee fusion using unconstrained and semiconstrained components: a report of 7 cases. J Arthroplasty, 2001. 16(6): p. 768-76

20. Wiedel, J.D., Salvage of infected total knee fusion: the last option. Clin Orthop Relat Res, 2002(404): p. 139-42.

21. Parvizi J, G.T., Proceedings of the International Consensus Meeting on Periprosthetic Joint Infection. . J Arthroplasty, 2014. 29 Suppl 1(2): p. 1-130.

22. Sanguineti, F., et al., Total knee arthroplasty with rotating-hinge Endo-Model prosthesis: clinical results in complex primary and revision surgery. Arch Orthop Trauma Surg, 2014. 134(11): p. 1601-7.

23. Bistolfi, A., et al., Results with 98 Endo-Modell rotating hinge prostheses for primary knee arthroplasty. Orthopedics, 2013. 36(6): p. e746-52.
SUBMISSION HISTORY

Submitted August 2, 2018

Reviewed August 18, 2018

Revised August 19, 2018

Accepted August 20, 2018

Published September 30, 2018

AUTHOR AFFILIATIONS

1 Jon E. Minter, DO

Arthritis \& Total Joint Specialists

3400-C Old Milton Parkway, Alpharetta, Georgia 30005

(Direct inquires to Jon Minter, MinterJE@gmail.com)

AUTHOR DISCLOSURES

The authors declare that there are no disclosures regarding the publication of this paper.

\section{COPYRIGHT \& OPEN ACCESS}

(C) 2018 Minter. All rights reserved.

Authors retain copyright and grant the journal right of first publication with the work. Reconstructive Review is an open access publication and follows the Creative Commons Attribution-NonCommercial CC BY-NC. This license allows anyone to download works, build upon the material, and share them with others for non-commercial purposes as long as they credit the senior author, Reconstructive Review, and the Joint Implant Surgery \& Research Foundation (JISRF). An example credit would be: "Courtesy of (senior author's name), Reconstructive Review, JISRF, Chagrin Falls, Ohio". 